\title{
Association of High-Risk Drinking with Metabolic Syndrome and Its Components in Elderly Korean Men: The Korean National Health and Nutrition Examination Survey 2010-2012
}

\author{
Ji-Hyun Kim', Jeong-Im Ha', Jae-Min Park'*, Ji Sun Lee', Ah-Leum Ahn', Eun-Jung Oh', Jae-Kyung Choi', \\ Hyuk-Jung Kweon', Dong-Yung Cho' \\ 'Department of Family Medicine, Konkuk University Medical Center, Seoul, Korea \\ ${ }^{2}$ Department of Family Medicine, Gangnam Severance Hospital, Yonsei University College of Medicine, Seoul, Korea
}

\section{See editorial commentary page on 209}

Background: Previous studies have examined the association between alcohol consumption and metabolic syndrome (MetS) in adults, but studies in the elderly are lacking. We examined the relationship between high-risk alcohol consumption and MetS in elderly Korean men using the Alcohol Use Disorders Identification Test (AUDIT) questionnaire from the 2010-2012 Korean National Health and Nutrition Examination Survey.

Methods: Among 25,534 subjects, 2,807 were men $>60$ years of age; after exclusions, we included 2,088 men in the final analysis. We categorized the study participants into three groups according to AUDIT score: low risk (0-7), intermediate risk (8-14), and high risk ( $\geq 15$ points).

Results: Among the study population, $17.0 \%$ of the men were high-risk drinkers, who had the highest mean waist circumference, systolic and diastolic blood pressure (BP), fasting plasma glucose (FPG), and triglyceride (TG) levels. The overall prevalence of MetS was $41.9 \%$ in the elderly men, and it was significantly higher in the group with high (48.3\%) versus low (31.9\%) AUDIT scores. The prevalence of MetS components (elevated BP, high FPG, high TG, and low high-density lipoprotein cholesterol) was associated with a high AUDIT score. The odds ratios (95\% confidence interval) of the high-risk group for MetS, elevated BP, and high TG were 1.40 (1.03-1.89), 1.82 (1.282.60), and 1.77 (1.30-2.41) after adjustment for confounding variables.

Conclusion: AUDIT score was correlated with most MetS components in elderly Korean men.

Keywords: Aged; Alcohol Drinking; Alcohol Use Disorders Identification Test; Metabolic Syndrome; Cross-Sectional Studies 


\section{INTRODUCTION}

Metabolic syndrome (MetS) is a cluster of metabolic abnormalities including abdominal obesity, elevated blood pressure (BP), dyslipidemia, and high fasting blood glucose. ${ }^{1)}$ MetS is related to type 2 diabetes mellitus, cardiovascular disease, cerebrovascular disease, and certain cancers. ${ }^{2-5)}$ It is also associated with increased all-cause mortality as well as cardiovascular mortality. ${ }^{6}$ With rapid socioeconomic growth and Westernized lifestyle habits, the prevalence of MetS is on the rise, and this increase threatens public health. ${ }^{7)}$

Korea is rapidly becoming an aging society, ${ }^{8,9)}$ and it is expected that by 2030 , the elderly population will have increased by $24.3 \% .^{9)}$ Because the prevalence of MetS is greater in older people, its risk is higher among the elderly than in the general population. ${ }^{10)}$ Several previous studies indicated that MetS increases the risk of all-cause mortality in the elderly. ${ }^{11,12)}$ Thus, preventing and managing MetS in the elderly are important from a public health perspective.

MetS is caused by both genetic and environmental factors (daily calorie intake, physical activity, household income, smoking, and alcohol consumption) ${ }^{13-15)}$ Because environmental factors can be controlled, appropriately managing them can contribute to managing MetS.

Hazardous drinking has been reported as an environmental cause of MetS, ${ }^{16,17)}$ and the Alcohol Use Disorders Identification Test (AUDIT) is widely used to screen for hazardous drinking. ${ }^{18)}$ This questionnaire assesses drinking quantity and patterns, and it can be used to identify high-risk drinkers who have related social or personal health problems. Previous studies have shown an association between alcohol consumption and MetS in adults, ${ }^{19)}$ but there is a lack of research on the relationship between high-risk alcohol consumption and MetS and its components in the elderly. Therefore, here we examined this relationship in elderly men using their AUDIT questionnaire responses from the 2010-2012 (fifth) Korean National Health and Nutrition Examination Survey (KNHANES).

\section{METHODS}

\section{Study Population}

We obtained the data used in this cross-sectional study from the KNHANES conducted in 2010-2012 by the Korea Centers for Disease Control and Prevention. The sampling units were households selected through stratified multistage probability sampling based on geographic region, sex, and age based on the registries. The study participants, civilian non-institutionalized South Koreans $>1$ year of age, were asked to answer the questionnaire, which was composed of a health interview survey, health behavior survey, health examination survey, and nutrition survey. All participants provided written informed consent and were given the right to refuse to participate according to the National Health Enhancement Act. Because all KNHANES data are publicly available, this study did not require institutional review board approval.

A total of 25,534 participants were surveyed in the 2010-2012
KNHANES; of them, 2,807 were elderly men aged $\geq 60$ years. We excluded men who had not fasted 8 hours prior to having their blood sampled ( $n=255)$ and those for whom data were missing $(n=464)$; we ultimately included 2,088 participants in the final analysis.

\section{Anthropometric and Laboratory Data}

The participants self-reported sociodemographic factors such as age, sex, education, income, and residence on the questionnaires. Trained staff measured body weight and height to the nearest $0.1 \mathrm{~kg}$ and $0.1 \mathrm{~cm}$, respectively, using standardized techniques and equipment, and we calculated body mass index by dividing weight by height squared ( $\mathrm{kg} /$ $\mathrm{m}^{2}$ ). Trained staff also measured BP in the right arm using a standard sphygmomanometer twice at a 5-minute interval and then averaged the values. Blood samples were obtained after overnight fasting. Fasting plasma glucose (FPG), total cholesterol, triglyceride (TG), and high-density lipoprotein cholesterol (HDL-C) levels were measured in a central, certified laboratory. A questionnaire based on the 24-hour recall method was used to collect data on the food items participants consumed during the previous 24 hours. The daily intake of energy and nutrients was calculated using Can-Pro Nutrient Intake Assessment software (Korean Nutrition Society, Seoul, Korea).

\section{Definition of Metabolic Syndrome, Alcohol Use Disorders Identification Test, and Lifestyle Factors}

We used the definitions of MetS and its components proposed by the National Cholesterol Education Program Adult Treatment Panel III ${ }^{20)}$ and the Korean Society for the Study of Obesity. ${ }^{21)}$ Specifically, we defined it as showing three or more of the following symptoms: (1) abdominal obesity (waist circumference $\geq 90 \mathrm{~cm}$ in men or $\geq 85 \mathrm{~cm}$ in women); (2) elevated blood pressure (systolic/diastolic BP [SBP/DBP] $\geq 130 / 85 \mathrm{~mm}$ Hg or taking antihypertensive medication); (3) impaired FPG ( $\geq 100 \mathrm{mg} / \mathrm{dL}$ ), diagnosis of diabetes mellitus, and/or taking insulin or a diabetes pill; (4) high plasma TG ( $\geq 150 \mathrm{mg} / \mathrm{dL}$ ); and (5) low HDL-C ( $<40 \mathrm{mg} / \mathrm{dL}$ in men or $<50 \mathrm{mg} / \mathrm{dL}$ in women).

The AUDIT questionnaire is used to identify high-risk drinkers, while AUDIT scores reflect the answers to 10 questions about recent alcohol use, alcohol dependence symptoms, and alcohol-related symptoms: ${ }^{22)}$ (1) drinking frequency; (2) usual drinking quantity; (3) frequency of high-risk drinking; (4) frequency of inability to stop drinking; (5) frequency of failure to do normally expected tasks; (6) frequency of needing a drink in the morning after a heavy drinking session; (7) frequency of feeling guilty after drinking; (8) frequency of inability to remember after drinking; (9) injury because of drinking; and (10) any suggestion by family members, friends, or doctors to cut down on drinking.

We categorized the study participants into three groups based on AUDIT score: 0-7, low risk; 8-14, intermediate risk; and $\geq 15$, high risk. These cut-off points were based on previous studies that screened participants for harmful drinking patterns. ${ }^{18,22)}$

We used other questionnaires to gather data regarding health-related lifestyle factors during the relevant time period. We estimated phys- 
ical activity using the Korean version of the International Physical Activity Questionnaire short form, ${ }^{23)}$ which categorizes regular exercisers as performing $\geq 20$ minutes of vigorous physical activity $\geq 3$ days a week or $\geq 30$ minutes of moderate physical activity $\geq 5$ days a week. We also defined current smokers as individuals who had smoked cigarettes during the relevant survey period.

\section{Statistical Analysis}

We applied sampling weights to each participant's data to ensure that the dataset represented the entire Korean population without biased estimates. We summarized participant characteristics based on their alcohol drinking patterns using weighted one-way analysis of variance for continuous variables and weighted chi-square tests for categorical variables. We also assessed the prevalence of MetS according to drinking patterns using the weighted chi-square test. We calculated the odds ratios (ORs) and 95\% confidence intervals (CIs) for MetS using multiple logistic regression analyses after we adjusted for confounding variables according to drinking patterns. We conducted all analyses using IBM SPSS ver. 23.0 (IBM Corp., Armonk, NY, USA) and considered values of $\mathrm{P}<0.05$ significant.

\section{RESULTS}

Table 1 shows the characteristics of the study population according to alcohol drinking pattern (AUDIT score); $17 \%$ of respondents were high-risk drinkers. The high-risk drinkers had the highest mean waist circumference, SBP, DBP, FPG, and TG.
Figure 1 shows the prevalence of MetS based on AUDIT score. The overall MetS prevalence was $41.9 \%$ in the elderly men and was significantly higher among the men with high rather than low AUDIT scores. By group, the prevalence in the low-, intermediate-, and high-risk drinking groups was $31.9 \%, 43.8 \%$, and $48.3 \%$, respectively.

Table 2 shows the prevalence of MetS components based on alcohol drinking pattern (AUDIT score). The prevalences of elevated BP, high FPG, high TG, and low HDL-C were associated with a high AUDIT score.

Table 3 presents the ORs and 95\% CIs based on AUDIT score. We adjusted the multiple logistic regression models for age, daily calorie

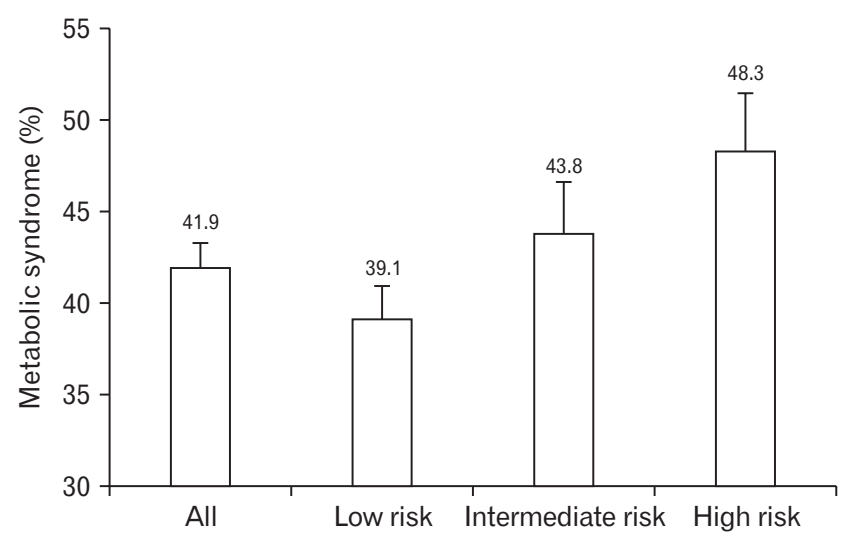

Figure 1. Prevalence of metabolic syndrome according to alcohol-drinking pattern (bars mean standard errors).

Table 1. Characteristics of study participants based on alcohol drinking pattern (Alcohol Use Disorders Identification Test score)

\begin{tabular}{|c|c|c|c|c|c|}
\hline Characteristic & All & Low risk (0-7) & Intermediate risk (8-14) & High risk ( $\geq 15$ ) & P-value \\
\hline Unweighted no. of participants & 2,088 & 1,258 & 474 & 356 & \\
\hline Age $(y)$ & $68.24 \pm 0.2$ & $69.2 \pm 0.2$ & $67.3 \pm 0.3$ & $66.5 \pm 0.3$ & $<0.001$ \\
\hline Body mass index $\left(\mathrm{kg} / \mathrm{m}^{2}\right)$ & $23.6 \pm 0.1$ & $23.4 \pm 0.1$ & $23.9 \pm 0.2$ & $23.8 \pm 0.2$ & 0.013 \\
\hline Waist circumference (cm) & $85.6 \pm 0.3$ & $84.8 \pm 0.3$ & $86.1 \pm 0.5$ & $87.0 \pm 0.6$ & 0.001 \\
\hline Systolic blood pressure (mm Hg) & $128.7 \pm 0.5$ & $127.5 \pm 0.6$ & $129.1 \pm 1.2$ & $132.1 \pm 1.4$ & 0.007 \\
\hline Diastolic blood pressure $(\mathrm{mm} \mathrm{Hg})$ & $76.8 \pm 0.3$ & $75.7 \pm 0.4$ & $77.8 \pm 0.6$ & $78.7 \pm 0.7$ & $<0.001$ \\
\hline Fasting plasma glucose (mg/dL) & $106.2 \pm 0.7$ & $104.3 \pm 0.8$ & $106.7 \pm 1.4$ & $111.2 \pm 1.9$ & 0.003 \\
\hline Total cholesterol (mg/dL) & $182.6 \pm 0.9$ & $183.2 \pm 1.0$ & $181.5 \pm 2.0$ & $182.0 \pm 2.2$ & 0.696 \\
\hline Triglyceride (mg/dL) & $142.8 \pm 2.6$ & $130.9 \pm 2.8$ & $158.2 \pm 6.8$ & $160.4 \pm 6.3$ & $<0.001$ \\
\hline High-density lipoprotein cholesterol (mg/dL) & $46.4 \pm 0.3$ & $44.2 \pm 0.4$ & $48.2 \pm 0.6$ & $50.6 \pm 0.8$ & $<0.001$ \\
\hline Hypertension (\%) & $56.6(1.3)$ & $53.1(1.7)$ & $60.1(3.0)$ & $63.3(3.0)$ & 0.012 \\
\hline Diabetes (\%) & $21.3(1.0)$ & $20.1(1.2)$ & $22.3(2.1)$ & $23.7(2.9)$ & 0.423 \\
\hline Calorie intake (kcal/d) & $2075 \pm 21$ & $2004 \pm 26$ & $2106 \pm 35$ & $2261 \pm 50$ & $<0.001$ \\
\hline Regular exercise (\%) & $20.2(1.2)$ & $19.1(1.5)$ & $22.1(2.3)$ & $21.5(2.5)$ & 0.442 \\
\hline Current smoker (\%) & $28.8(1.2)$ & $24.5(1.4)$ & $32.9(2.6)$ & $37.0(3.0)$ & $<0.001$ \\
\hline Household income (US \$/mo) & $2799 \pm 175$ & $2494 \pm 111$ & $3144 \pm 226$ & $3318 \pm 645$ & 0.182 \\
\hline Residence in a rural area (\%) & $30.0(2.5)$ & $30.5(2.7)$ & $28.3(3.3)$ & $30.4(3.8)$ & 0.774 \\
\hline Education level (\%) & & & & & 0.149 \\
\hline$\leq 6 \mathrm{y}$ & $41.9(1.7)$ & $41.9(1.9)$ & $41.0(3.0)$ & $42.9(3.3)$ & \\
\hline $7-9 y$ & $19.1(1.3)$ & $18.5(1.5)$ & $22.3(2.6)$ & $16.9(2.4)$ & \\
\hline $10-12 y$ & $25.6(1.3)$ & $24.1(1.5)$ & $26.3(2.7)$ & $29.1(2.9)$ & \\
\hline$>13 y$ & $13.4(1.1)$ & $15.4(1.4)$ & $10.3(1.8)$ & $11.0(2.5)$ & \\
\hline
\end{tabular}

Values are presented as mean \pm standard error or \% (standard error). P-values were obtained using weighted one-way analysis of variance for continuous variables and weighted chi-square tests for categorical variables. US $\$ 1=1,000$ Korean won. 
Table 2. Prevalence of metabolic syndrome and its components based on alcohol drinking pattern (Alcohol Use Disorders Identification Test score)

\begin{tabular}{|c|c|c|c|c|c|}
\hline Variable & All & Low risk (0-7) & Intermediate risk (8-14) & High risk ( $\geq 15)$ & P-value \\
\hline Metabolic syndrome (\%) & $41.9(1.4)$ & $39.1(1.8)$ & $43.8(2.8)$ & $48.3(3.2)$ & 0.026 \\
\hline Abdominal obesity (\%) & $31.0(1.3)$ & $28.5(1.6)$ & $33.5(2.7)$ & $35.5(3.3)$ & 0.070 \\
\hline Elevated blood pressure (\%) & $67.6(1.2)$ & $64.1(1.7)$ & $70.2(2.9)$ & $75.0(2.6)$ & 0.006 \\
\hline High fasting plasma glucose (\%) & $50.6(1.3)$ & $47.8(1.7)$ & $52.7(2.8)$ & $56.6(3.1)$ & 0.030 \\
\hline High triglyceride (\%) & $38.8(1.4)$ & $33.6(1.7)$ & $44.0(2.9)$ & $48.5(3.1)$ & $<0.001$ \\
\hline Low high-density lipoprotein cholesterol (\%) & $39.7(1.3)$ & $46.1(1.8)$ & $34.3(2.4)$ & $26.8(2.7)$ & $<0.001$ \\
\hline
\end{tabular}

Values are presented as \% (standard error). P-values were obtained using a weighted chi-square test.

Table 3. Multivariate-adjusted odds ratios and 95\% confidence intervals for metabolic syndrome and its components based on alcohol drinking pattern (Alcohol Use Disorders Identification Test score)

\begin{tabular}{lccc}
\hline \multicolumn{1}{c}{ Variable } & Low risk (0-7) & Intermediate risk (8-14) & High risk $(\geq 15)$ \\
\hline MetS & 1 & $1.23(0.94-1.61)$ & $1.40(1.03-1.89)$ \\
Abdominal obesity & 1 & $1.37(1.03-1.83)$ & $1.41(1.02-2.00)$ \\
Elevated BP & 1 & $1.27(0.91-1.78)$ & $1.82(1.28-2.60)$ \\
High FPG & 1 & $1.12(0.86-1.45)$ & $1.29(0.97-1.73)$ \\
High TG & 1 & $1.54(1.17-2.03)$ & $1.77(1.30-2.41)$ \\
Low HDL-C & 1 & $0.64(0.49-0.83)$ & $0.37(0.27-0.51)$ \\
\hline
\end{tabular}

Values are presented as odds ratio (95\% confidence interval). Odds ratios for MetS, abdominal obesity, elevated BP, high FPG, high TG, and low HDL-C were determined using multivariate logistic regression analysis after the adjustment for age, daily calorie intake, exercise status, smoking status, monthly household income, residential area, and education level.

Mets, metabolic syndrome; BP, blood pressure; FPG, fasting plasma glucose; TG, triglyceride; HDL-C, high-density lipoprotein cholesterol.

intake, exercise status, smoking status, monthly household income, residential area, and education level. Compared to men in the low-risk group, those in the high-risk group were at significantly higher risk of MetS and its components except for high FPG and low HDL-C. The ORs (95\% CI) of the high-risk group for MetS, elevated BP, and a high TG were 1.40 (1.03-1.89), 1.82 (1.28-2.60), and 1.77 (1.30-2.41) after the adjustment for confounding variables.

\section{DISCUSSION}

Here we found that high-risk drinking was associated with a higher risk of MetS in elderly Korean men. Our findings are in agreement with the results of previous studies that showed that AUDIT score was related to MetS in adult populations, and our results indicate that this relationship applies even when confined to elderly individuals; we analyzed data from 2,088 elderly men and found that MetS and most of its components were significantly associated with AUDIT scores.

Korea is rapidly becoming an aging society, and MetS is more prevalent in the elderly; ${ }^{10)}$ therefore, a study of the relationship between high-risk alcohol and MetS in the elderly population was necessary. In this study, the prevalence of MetS in elderly Korean men was $41.9 \%$, similar to the findings from previous studies and higher than the prevalence among the elderly in Europe and the United States. ${ }^{24,25)}$ As previously mentioned, MetS is strongly associated with type 2 diabetes mellitus, cardiovascular disease, cerebrovascular disease, certain cancers, and increased mortality; therefore, the management of MetS and its components is important among young and elderly adults. Here we showed that it is important to manage high-risk drinking, an adjust- able factor in MetS management, in elderly populations.

MetS is related to lifestyle factors including dietary intake, ${ }^{15)}$ exercise, ${ }^{14)}$ and smoking. ${ }^{14)}$ It is also associated with socioeconomic status variables such as household income ${ }^{13,14)}$ and education level. ${ }^{13,14)}$ Thus, we included these lifestyle factors and socioeconomic status in the multiple logistic regression analyses to control for them.

The effects of high-risk drinking on metabolic abnormalities were addressed in previous studies. Heavy alcohol consumption is associated with abdominal obesity, ${ }^{26)}$ type 2 diabetes, ${ }^{27)}$ hypertension, ${ }^{28)}$ and dyslipidemia. ${ }^{29)}$ Although there are previous studies on the relationship between alcohol consumption and MetS, ${ }^{16,17)}$ our research contributes to our understanding of alcohol consumption related to MetS in the elderly. Shin et al. ${ }^{30)}$ reported that unhealthy drinking patterns were significantly associated with MetS in older adults, but although the participants in that study were $>50$ years old, they were confined to only one urban area in South Korea; for that reason, the study could not explore associations in the general Korean elderly population. Our study showed that this association between high-risk drinking and MetS applies specifically to the elderly, who have a high prevalence of MetS. In addition, because our study was based on KNHANES data, our findings are representative of the entire national population. Our study also has the advantage of predicting the prevalence of MetS in the elderly.

There are some limitations of this study that should be considered when interpreting its results. First, it was a cross-sectional study; as such, we could not draw conclusions about causality between alcohol consumption and MetS prevalence. Second, the study was confined to elderly Korean males; thus, our findings may be difficult to generalize 
to other ethnic groups and women. Third, we did not analyze data subdivided by age. However, to minimize this limitation, we included age as a confounding variable in the multiple logistic regression analysis. Fourth, we used self-reported information on alcohol consumption; thus, there might have been underestimation of alcohol consumption, including memory inaccuracies. Finally, the data we used did not include beverage types; thus, we could not tell the effects of beverage type on MetS. Nevertheless, to the best of our knowledge, this is the first national population-based study to show a link between alcohol consumption and MetS in the elderly.

In conclusion, AUDIT score related to MetS and most of its components in elderly Korean men. Managing MetS and its components in the elderly is important for national health policies and primary care, and high-risk drinking is an adjustable factor that is important for managing the condition. Further research is warranted to investigate the effect of alcohol on health in old age.

\section{CONFLICT OF INTEREST}

No potential conflict of interest relevant to this article was reported.

\section{REFERENCES}

1. Eckel RH, Grundy SM, Zimmet PZ. The metabolic syndrome. Lancet 2005;365:1415-28.

2. Ford ES. Risks for all-cause mortality, cardiovascular disease, and diabetes associated with the metabolic syndrome: a summary of the evidence. Diabetes Care 2005;28:1769-78.

3. Gami AS, Witt BJ, Howard DE, Erwin PJ, Gami LA, Somers VK, et al. Metabolic syndrome and risk of incident cardiovascular events and death: a systematic review and meta-analysis of longitudinal studies. J Am Coll Cardiol 2007;49:403-14.

4. Isomaa B, Almgren P, Tuomi T, Forsen B, Lahti K, Nissen M, et al. Cardiovascular morbidity and mortality associated with the metabolic syndrome. Diabetes Care 2001;24:683-9.

5. Valantine H, Rickenbacker P, Kemna M, Hunt S, Chen YD, Reaven G, et al. Metabolic abnormalities characteristic of dysmetabolic syndrome predict the development of transplant coronary artery disease: a prospective study. Circulation 2001;103:2144-52.

6. Trevisan M, Liu J, Bahsas FB, Menotti A. Syndrome X and mortality: a population-based study: Risk Factor and Life Expectancy Research Group. Am J Epidemiol 1998;148:958-66.

7. Lim S, Shin H, Song JH, Kwak SH, Kang SM, Yoon JW, et al. Increasing prevalence of metabolic syndrome in Korea: the Korean National Health and Nutrition Examination Survey for 1998-2007. Diabetes Care 2011;34:1323-8.

8. The Korean Society for Preventive Medicine. Preventive medicine and public health. 2nd ed. Seoul: Gyechuk Munwhasa; 2013.

9. Statistics Korea. Population trends and projections of the World and Korea [Internet]. Daejeon: Statistics Korea; 2015 [cited 2016 Feb 20]. Available from: http://kostat.go.kr/portal/eng/pressReleases/8/8/index.board.

10. Dominguez LJ, Barbagallo M. The cardiometabolic syndrome and sar- copenic obesity in older persons. J Cardiometab Syndr 2007;2:183-9.

11. Malyutina S, Bobak M, Kurilovitch S, Gafarov V, Simonova G, Nikitin Y, et al. Relation between heavy and binge drinking and all-cause and cardiovascular mortality in Novosibirsk, Russia: a prospective cohort study. Lancet 2002;360:1448-54.

12. Ghaem Maralani H, Tai BC, Wong TY, Tai ES, Li J, Wang JJ, et al. Metabolic syndrome and mortality in the elderly: a time-dependent association. Diabetes Res Clin Pract 2013;99:209-16.

13. Loucks EB, Rehkopf DH, Thurston RC, Kawachi I. Socioeconomic disparities in metabolic syndrome differ by gender: evidence from NHANES III. Ann Epidemiol 2007;17:19-26.

14. Park YW, Zhu S, Palaniappan L, Heshka S, Carnethon MR, Heymsfield SB. The metabolic syndrome: prevalence and associated risk factor findings in the US population from the Third National Health and Nutrition Examination Survey, 1988-1994. Arch Intern Med 2003;163:42736.

15. McKeown NM, Meigs JB, Liu S, Saltzman E, Wilson PW, Jacques PF. Carbohydrate nutrition, insulin resistance, and the prevalence of the metabolic syndrome in the Framingham Offspring Cohort. Diabetes Care 2004;27:538-46.

16. Baik I, Shin C. Prospective study of alcohol consumption and metabolic syndrome. Am J Clin Nutr 2008;87:1455-63.

17. Fan JG, Cai XB, Li L, Li XJ, Dai F, Zhu J. Alcohol consumption and metabolic syndrome among Shanghai adults: a randomized multistage stratified cluster sampling investigation. World J Gastroenterol 2008; 14:2418-24.

18. Skipsey K, Burleson JA, Kranzler HR. Utility of the AUDIT for identification of hazardous or harmful drinking in drug-dependent patients. Drug Alcohol Depend 1997;45:157-63.

19. Lee KW, Park BJ, Kang HT, Lee YJ. Alcohol-drinking patterns and metabolic syndrome risk: the 2007 Korean National Health and Nutrition Examination Survey. Alcohol 2011;45:499-505.

20. National Cholesterol Education Program (NCEP) Expert Panel on Detection, Evaluation, and Treatment of High Blood Cholesterol in Adults (Adult Treatment Panel III). Third report of the National Cholesterol Education Program (NCEP) Expert Panel on Detection, Evaluation, and Treatment of High Blood Cholesterol in Adults (Adult Treatment Panel III) final report. Circulation 2002;106:3143-421.

21. Lee SY, Park HS, Kim DJ, Han JH, Kim SM, Cho GJ, et al. Appropriate waist circumference cutoff points for central obesity in Korean adults. Diabetes Res Clin Pract 2007;75:72-80.

22. Babor TF, Higgins-Biddle JC, Saunders JB, Monteiro MG; World Health Organization. AUDIT: the Alcohol Use Disorders Identification Test: guidelines for use in primary health care. 2nd ed. Geneva: World Health Organization; 2001.

23. Oh JY, Yang YJ, Kim BS, Kang JH. Validity and reliability of Korean version of International Physical Activity Questionnaire (IPAQ) Short Form. J Korean Acad Fam Med 2007;28:532-41.

24. Guize L, Thomas F, Pannier B, Bean K, Danchin N, Benetos A. Metabolic syndrome: prevalence, risk factors and mortality in a French population of 62000 subjects. Bull Acad Natl Med 2006;190:685-97.

25. Ford ES, Giles WH, Dietz WH. Prevalence of the metabolic syndrome among US adults: findings from the third National Health and Nutrition Examination Survey. JAMA 2002;287:356-9.

26. Yokoyama H, Hiroshi H, Ohgo H, Hibi T, Saito I. Effects of excessive 
ethanol consumption on the diagnosis of the metabolic syndrome using its clinical diagnostic criteria. Intern Med 2007;46:1345-52.

27. Baliunas DO, Taylor BJ, Irving H, Roerecke M, Patra J, Mohapatra S, et al. Alcohol as a risk factor for type 2 diabetes: a systematic review and meta-analysis. Diabetes Care 2009;32:2123-32.

28. Briasoulis A, Agarwal V, Messerli FH. Alcohol consumption and the risk of hypertension in men and women: a systematic review and meta-analysis. J Clin Hypertens (Greenwich) 2012;14:792-8.
29. Wang Z, Yao T, Song Z. Chronic alcohol consumption disrupted cholesterol homeostasis in rats: down-regulation of low-density lipoprotein receptor and enhancement of cholesterol biosynthesis pathway in the liver. Alcohol Clin Exp Res 2010;34:471-8.

30. Shin MH, Kweon SS, Choi JS, Rhee JA, Nam HS, Jeong SK, et al. Average volume of alcohol consumed, drinking patterns, and metabolic syndrome in older Korean adults. J Epidemiol 2013;23:122-31. 